\title{
Erratum to: Penicillin G acylase from Achromobacter sp. CCM 4824
}

\section{An efficient biocatalyst for syntheses of beta-lactam antibiotics under conditions employed in large-scale processes}

\author{
Stanislav Bečka • Václav Štěpánek • Rajasekar W. Vyasarayani • Michal Grulich • \\ Jaroslav Maršálek • Kamila Plháčková • Marie Dobišová • Helena Marešová • \\ Martina Plačková • Renáta Valešová • Andrea Palyzová • Anupama Datla • \\ Trupti K. Ashar • Pavel Kyslík
}

Published online: 3 October 2014

(C) Springer-Verlag Berlin Heidelberg 2014

\section{Erratum to: Appl Microbiol Biotechnol (2014) 98:1195-1203 DOI 10.1007/s00253-013-4945-3}

The above mentioned article has been published online on 15th May 2013 in Appl Microbiol Biotechnol. The authors would like to correct the paragraph on the strain CCM 4824 origin (Introduction, p. 1196, left column - the last paragraph) published as "We described a novel penicillin G acylase (NPGA) from soil isolate Achromobacter sp. CCM 4824 (Plháčková et al. 2003; Škrob et al. 2003)".

The corrected presentation should be "We described a novel penicillin G acylase (NPGA) from a mutant of soil isolate Achromobacter sp. CCM 4824 (Plháčková et al. 2003; Škrob et al. 2003; Kyslík et al. 2011)”.

The online version of the original article can be found at http://dx.doi.org/ 10.1007/s00253-013-4945-3.

S. Bečka $\cdot$ V. Štěpánek $\cdot$ M. Grulich $\cdot J$. Maršálek $\cdot$ K. Plháčková $\cdot$

M. Dobišová $\cdot H$. Marešová $\cdot M$. Plačková $\cdot R$. Valešová $\cdot$

A. Palyzová $\cdot$ P. Kyslík $(\bowtie)$

Laboratory of Enzyme Technology, Institute of Microbiology

ASCR, v.v.i, Prague, Czech Republic

e-mail:kyslik@biomed.cas.cz

R. W. Vyasarayani $\cdot$ A. Datla $\cdot$ T. K. Ashar

Fermenta Biotech Ltd, Thane, India 